\title{
Emergent GUP from Modified Hawking Radiation in Einstein-NED Theory
}

\author{
S. Hamid Mehdipour ${ }^{1, *}$ \\ ${ }^{1}$ Department of Physics, College of Basic Sciences, Lahijan Branch, \\ Islamic Azad University, P. O. Box 1616, Lahijan, Iran
}

(Dated: November 4, 2019)

\begin{abstract}
We present a general procedure for constructing exact black hole $(\mathrm{BH})$ solutions with the magnetic charge in the context of nonlinear electrodynamics (NED) theory as well as in the coherent state approach to noncommutative geometry (NCG). In this framework, the Lagrangian density for the noncommutative Hayward BH is obtained and the weak energy condition (WEC) is satisfied. The noncommutative Hayward solution depends on two kind of charges which for the vanishing of them yields the Schwarzschild solution. Moreover, in order to find a link between the BH evaporation and uncertainty relations, we may calculate the Hawking temperature and find the effect of the Lagrangian density of BHs on the Hawking radiation. Therefore, a generalized uncertainty principle (GUP) emerges from the modified Hawking temperature in Einstein-NED theory. The origin of this GUP is the combined influence of a nonlinear magnetic source and an intrinsic property of the manifold associated with a fictitious charge. Finally, we find that there is an upper bound on the Lagrangian uncertainty of the BHs which are sourced by the NED field and/or the fictitious charge.

PACS numbers: 04.70.Dy, 04.20.Jb, 02.40.Gh, 04.20.Dw
\end{abstract}

Keywords: Regular Black Holes; Nonlinear Electrodynamics; Noncommutative Geometry; Hawking Temperature; Uncertainty Relations.

*Electronic address: mehdipour@liau.ac.ir 


\section{INTRODUCTION}

It has been shown that the physical source of a regular black hole (RBH) can be interpreted as the gravitational field of a nonlinear electrodynamics (NED) [1 6]. NED models emerge from the low-energy effective limit in particular approaches to string/M-theories [7 9]. There are two important purposes in a NED theory. One is to take into account electromagnetic field and particles in the frame of a physical source and the other is to ignore allowing physical quantities turn into infinite. A same process is attained by a physically reliable NED model coupled to gravity so that regular spherically symmetric electrically charged solutions satisfy the weak energy condition (WEC) and contain an inescapable deSitter centre of the nonlinear electrodynamic source [10]. The metric and curvature invariants of charged RBHs, incompatible with Reissner-Nordström (R-N) BHs, are regular everywhere. The Bardeen $\mathrm{BH}$ is the first $\mathrm{RBH}$ model in general relativity (GR) which has been proposed by the pioneering work of Bardeen in 1968 [11]. This BH has an event horizon and a deSitter-like feature inside its horizon which does not violate the WEC. A bit later, Pelliger and Torrence [1] obtained a general static, spherically symmetric solution with an electric charge in GR coupled to the NED with a gauge-invariant Lagrangian. Later, in 1976, Bronnikov and Shikin [2] proved in a general form that if this NED has a Maxwell weak field limit, then the above solution cannot have a regular centre (see also [3] ). In 2001, Bronnikov [12] extended the solution to include radial magnetic field and showed that only purely magnetic configurations can have a regular centre, while the regular metric "profile" in this case can be arbitrary. The Hayward solution is another well-known kind of regular spacetime wherein its static region is Bardeen-like while the dynamic regions are Vaidya-

like [13]. Ayon-Beato and Garcia reinterpreted the Bardeen BH as a magnetic solution to Einstein equations with the NED [14]. The other solutions of Einstein-NED theory have also been reported in [15 17]. There have been a great number of studies concerning the combined Einstein and NED equations in the literature [18 29].

The issue of central singularity in BHs is widely believed to be an unavoidable prediction of GR [30]. However, various phenomenological approaches have been considered in the literature for resolving the problem via a regular centre [10]. In a noncommutative geometry (NCG) inspired model (for a review see [31]), a point particle in a noncommutative spacetime is no longer characterized by a Dirac-delta function distribution, but will be described 
as a smeared particle by a Gaussian distribution of minimal width $\sqrt{\theta}$, beyond which coordinate resolution is obscure. Therefore, the Einstein tensor in gravity field equations stays unchanged however the energy-momentum tensor takes a new form. As a striking result of this noncommutativity model, the curvature singularity at the origin of BHs is removed. Instead of the curvature singularity, a regular deSitter vacuum state will appear concerning the effect of the strong quantum fluctuations at short distances. In fact, a noncommutative $\mathrm{BH}$ is a combination of the deSitter core around the origin with the ordinary metric of the $\mathrm{BH}$ far away from the origin. So, the existence of a deSitter core in the centre of a BH prevents its collapse into a singular state, and an ordinary metric at large distances is recovered where the behavior of the minimal length is not influential.

On the other hand, in recent years, due to various conceptual and technical reasons, it has been suggested that there may exist corrections to the Heisenberg uncertainty principle (HUP) which could be important at extreme scales, i.e. in the ultraviolet (UV) and in the infrared (IR) scales [32]. In the UV scale, the incorporation of gravity in quantum field theory yields effectively a cutoff in the high energy regime, i.e. a minimal length scale of the order of the Planck length, $l_{P} \approx 10^{-35} \mathrm{~m}$. Studies on string collisions at Planckian energies and through a renormalization group type of analysis lead to the modification of the HUP, i.e. the generalized uncertainty principle (GUP) [33] (for a review, see [34]). The thermodynamics of Schwarzschild and R-N BHs using the GUP was studied in [35]. The exact expressions for the mass-temperature relation, heat capacity and entropy were obtained. They revealed that the expression for the entropy is the well known area theorem in terms of the horizon area up to leading order corrections from the GUP. They also found that the the entropy has a singularity at zero corrected horizon area that refers to a critical mass which is less than the remnant mass at which the $\mathrm{BH}$ evaporation process halts. For a review of the various roads to the remnant scenario and their challenges for ameliorating the $\mathrm{BH}$ information loss problem [36], see [37].

The whole problems concerning the $\mathrm{BH}$ evaporation [38] are mostly owing to a failure of the semiclassical explanation under the condition that the $\mathrm{BH}$ mass should be extremely larger than the Planck mass. When the decay continues and the BH mass reduces, this condition ultimately can no longer be true and a theory of quantum gravity (QG) must be applied (for a review, see [39]). Further, Parikh and Wilczek [40] found that Hawking radiation can be extracted from the null geodesic method by considering the backreaction 
effects. They calculated a leading correction to the probability of massless particles tunneling across the horizon. The tunneling process illustrates an extended emitted spectrum that is not definitely thermal but is consistent with an underlying unitary quantum theory. In fact, the emission rate deviates from the pure thermal spectrum and results in unitarity; so that the conservation of information is supported [41]. There is another well-known tunneling approach for exploring the Hawking radiation as a quantum tunneling effect that is the Hamilton-Jacobi strategy [42]. Many authors considered alternative derivations to compute the Hawking radiation and also investigated the tunneling of various particles to obtain thermodynamical quantities such as the temperature and entropy for different kind of BHs [43].

Applying the GUP modified entropy to the entropic gravity scenario proposed by Verlinde [44], suggesting that the gravity is originally an entropic force, exhibits that the resulting gravity force law includes sub-leading order correction terms that is dependent on the Planck constant [45]. Such modification in the area law of the BH entropy could lead to a modified Newton's law of gravitation and could have observable consequences at length scales much larger than the Planck scale [46].

Recently, Feng et al. [47] have demonstrated that the dimensionless parameter in the GUP can be constrained by the gravitational wave event GW150914 discovered by the LIGO Scientific and Virgo Collaborations [48]. They have derived the energy-momentum dispersion relation and the difference between the propagation speed of gravitons and the speed of light using the HUP and the data of gravitational wave event. Moreover, they have obtained the upper limits of the GUP parameters based on the modified speed of gravitons. As another example of possible experimental signatures of modified uncertainty principle, Mureika [49] proposed an extended uncertainty principle that allows for large scale modifications to gravity. This would have an impact on the attributes of most known supermassive BHs, and therefore could be observed by the Event Horizon Telescope [50] or other future collaborations.

Here it is worth noting that there are also some comments on opposite ideas on GUP. For example, GUP measurements could have an effect on the violation of fundamental symmetries, like CPT and Lorentz invariance in the framework of the standard model extension [51]. The basic reason for this problem, as mentioned above, is that QG corrections such as those of the GUP serve no purpose in the context of Hawking radiation. It was already 
evident in the earliest literature [52] that the process of Hawking emission is only valid for semiclassically large BHs. In this case, such corrections would be undetectable leading-order quantum effects, is debatable to begin with. The same requirement of large BHs dooms the idea that anything can be learned about the end point of $\mathrm{BH}$ evaporation in this program. The legitimacy of BH thermodynamics has broken down long before the alleged remnants have been identified. In fact, any method that does predict the existence of remnants should be viewed upon with a large dose of skepticism.

Nevertheless, the application of the GUP to BH thermodynamics has attracted considerable attention and leads to significant modifications to the emission process, particularly at the final stages of the evaporation [53]. It is worth mentioning, the thermodynamics of BHs is thought to be the connection between $\mathrm{BH}$ physics and quantum theory. For instance, in 2001, Adler et al. [54] have argued that in opposition to standard viewpoint, the GUP may prevent small BHs from total disappearing in exactly the same way that the HUP obstructs the hydrogen atom from total collapse. They applied the GUP approach in an alternative heuristic derivation of the Hawking radiation to find a corrected $\mathrm{BH}$ temperature. In this heuristic method, the Hawking temperature can be achieved by the utilization of the HUP and general properties of BHs [55]. Many authors considered various problems in this framework, e.g., see [56].

Several studies in string theory and NCG give a particular type of correction to the HUP and thus propose the appearance of a finite limit to the possible resolution of distances at extreme scales [57]. Indeed, because of the appearance of extreme energies at short distances of a noncommutative manifold, the influences of manifold quantum fluctuations turn into noticeable and prevent any measurements to determine a particle position with a preciseness greater than an intrinsic length scale which can be understood as an example of locality violation at the Planck length [58]. It is interesting to note that the methodology of the NCG effectively represents itself as an equivalent description of the nonlocal field theory discussed in Ref. [59]. It can be inferred from BH physics that any theory of QG should have an intrinsic minimum length of the order of Planck length [60]. There are some other methods to investigate the QG effects of BHs that have been studied in the literature [61].

On the basis of the noncommutativity, the extracting of metrics for noncommutative BHs is identified with the possible running of the minimal observable length in GR. Recently, we have analysed the final stages of the $\mathrm{BH}$ evaporation for the noncommutative Bardeen 
and Hayward solutions [62, 63]. The results showed that the behavior of Hawking radiation changes considerably at the small radii regime such that the $\mathrm{BH}$ does not evaporate completely, but a stable BH remnant will be remained at the final phase of the evaporation. In this paper, we are going to find the Lagrangian density of a term depending nonlinearly on the electromagnetic field tensor for a family of spherically symmetric, static, charged $\mathrm{RBH}$ metrics. We obtain the Hawking temperature of the noncommutative Hayward BH and use its corrected temperature to derive a modified uncertainty relation including the Lagrangian density in exactly the same way that the GUP creates a modified Hawking temperature.

This paper is organized as follows. In Sec. III, we describe the nonlinear electromagnetic field equations in regular spacetimes. The noncommutative effects on the spacetime of Hayward are investigated in Sec. III. The resulting metric in three possible causal structures is analyzed and its WEC is examined. In Sec. IV], we determine the Hawking temperature of the noncommutative Hayward $\mathrm{BH}$ and then, in order to find the effect of the Lagrangian density on the Hawking radiation, we speak of a modified uncertainty relation for radiated photons that is derived from the HUP. Thus, a rough estimate between the Lagrangian density of RBHs and uncertainty relations is revealed. Finally, our results are briefly presented in Sec. V. Throughout the paper, Greek indices run from 0 to 3 and we use natural units with the following definitions: $\hbar=c=G=k_{B}=1$.

\section{NED FIELD COUPLED WITH GR}

Many configurations of NED coupled to GR now exist in the literature (for a brief review see [64]). In this section, we investigate the most general form of a spherically symmetric, static, charged RBH metrics in GR coupled to a NED. We start with the action describing the dynamics of a self-gravitating NED field in GR

$$
S=\frac{1}{16 \pi} \int \sqrt{-\mathrm{g}} d^{4} x(R-L(F))
$$

where $R$ is the curvature scalar with respect to the line element $\mathrm{g}_{\alpha \beta}$, and the Lagrangian density $L(F)$ is an arbitrary nonlinear function of the Lorentz invariant, $F=F_{\alpha \beta} F^{\alpha \beta}$, where $F_{\alpha \beta}=\partial_{\alpha} A_{\beta}-\partial_{\beta} A_{\alpha}$ is the electromagnetic field ( $A_{\alpha}$ is the 4-vector potential). It

should be noted that the Lagrangian density approaches to Maxwell asymptotic at weak electromagnetic fields, i.e. $L(F) \rightarrow F$, and $L_{F} \equiv \frac{d L}{d F} \rightarrow 1$ as $F \rightarrow 0$. 
The tensor field $F_{\alpha \beta}$ satisfies equations

$$
\begin{gathered}
\nabla_{\alpha}\left(L_{F} F^{\alpha \beta}\right)=0, \\
\nabla_{\alpha}{ }^{*} F^{\alpha \beta}=0,
\end{gathered}
$$

where the asterisk refers to the Hodge dual. The stress-energy tensor $T_{\alpha \beta}$ can be found by differentiating the action $S$ with respect to the metric tensor $\mathrm{g}_{\alpha \beta}$ as follows

$$
T_{\alpha \beta}=\frac{1}{4 \pi}\left(L_{F} F_{\gamma \alpha} F_{\beta}^{\gamma}-\frac{1}{4} \mathrm{~g}_{\alpha \beta} L\right) .
$$

The Einstein equations of motion are given by

$$
R_{\alpha \beta}-\frac{1}{2} \mathrm{~g}_{\alpha \beta} R=2\left(L_{F} F_{\gamma \alpha} F_{\beta}^{\gamma}-\frac{1}{4} \mathrm{~g}_{\alpha \beta} L\right) .
$$

Here, we consider a general ansatz of the BH solution having the static spherical symmetric configuration with the nonlinear magnetic charge

$$
\left\{\begin{array}{l}
d s^{2}=N(r) d t^{2}-N^{-1}(r) d r^{2}-r^{2}\left(d \vartheta^{2}+\sin ^{2} \vartheta d \phi^{2}\right), \\
A=e_{m} \cos \vartheta d \phi
\end{array}\right.
$$

where $e_{m}$ is the total magnetic charge carried by the $\mathrm{BH}$ related to the value of Hayward's free parameter $g$ (given in length unit). The value of $g$ is limited to the positive number, because the $\mathrm{BH}$ solution only exists at the positive value. The metric function $N(r)$ is defined by the relation

$$
N(r)=1-\frac{2 m(r)}{r}
$$

Einstein's field equations may be simplified to these independent forms

$$
\begin{gathered}
\frac{d^{2} N}{d r^{2}}-\frac{2}{r^{2}}(N-1)-\frac{4 e_{m}^{4}}{r^{4}} L_{F}=0, \\
\frac{1}{r} \frac{d N}{d r}+\frac{N-1}{r^{2}}+\frac{L}{2}=0 .
\end{gathered}
$$

It can be seen that Eq. (8) is satisfied for any given metric function (17), arising from the most general form of a static spherically symmetric solution with a magnetic charge. Hence, using Eqs. (7) and (9), the mass distribution function can be written in the form

$$
m(r)=\frac{1}{4} \int^{r} L\left(F\left(r^{\prime}\right)\right) r^{\prime 2} d r^{\prime}+C
$$


where $C$ is an integration constant. In order to obtain $m(r)$, it should be emphasized that as the equations of motion are too complicated to solve analytically for dyonic charges, the situation turns into much simpler for either magnetic or electric charges. In this paper, we are going to construct exact $\mathrm{BH}$ solutions with the magnetic charge because in the NED, the reason for regularity comes from the magnetic charge. In a purely magnetic configuration, $F_{\alpha \beta}$ is zero except for $F_{\vartheta \phi}$, that is

$$
F_{\vartheta \phi}=e_{m} \sin \vartheta
$$

Thus, using Eq. (2), we find the square of the field strength as

$$
F=\frac{2 e_{m}^{2}}{r^{4}}
$$

The lagrangian density as a function of $r$, i.e. $L=\frac{4}{r^{2}} \frac{d m}{d r}$, can be derived freely by selecting a physically appropriate mass function to create static solutions including magnetic charges. The ADM mass for a charged RBH has two terms, one from the Schwarzschild mass and the other from the nonlinear interactions between the graviton and the nonlinear photon. The latter is not possible for a linear Maxwell field. It is easily seen that the condition for the ADM mass at infinity leads to $m(r \rightarrow \infty)=M=C$ (or $L=0$ ), and the Schwarzschild $\mathrm{BH}$ is recovered as expected, which implies the solution of vacuum Einstein equations. In addition, for $m(r)=M-\frac{e_{m}^{2}}{2 r}$ (or $\left.L=F\right)$, which implies the solution of Einstein-Maxwell theories, we recover a magnetically charged $\mathrm{R}-\mathrm{N}$ BH.

We remind that the causal structures of RBHs are similar to the R-N BH, but with a deSitter centre instead of the singularity at $r=0$. The breakdown of the classical picture takes place only in a limited region around the origin, the region where gravity switches to a repulsive quantum interaction. This is notably different from the standard cases such as the Schwarzschild and R-N BHs which in general have a singularity at the origin.

In NED, no singularity exists in a RBH. This is because the source of RBHs is the nonlinear electrodynamic charge which differs from the Schwarzschild case that has a mass shrinking to the singularity as a result of losing mass by the emission of Hawking radiation. The early stage of the Hawking evaporation process for the RBH is similar to the Schwarzschild case, while for the late stage it is wholly disparate. The Schwarzschild BH loses energy by the thermal emission, with a gradual reduction in its mass and a growth on its temperature. The occurrence of a divergent behavior of the Hawking temperature is 
indeed due to the curvature singularity at the origin of the Schwarzschild manifold. However, one can expect that the evaporating $\mathrm{BH}$ will experience a Plank phase of the spacetime manifold in the vicinity of the origin; so that the evaporation is dramatically disturbed by strong quantum gravitational fluctuations in which a QG theory must be employed.

\section{NONCOMMUTATIVE HAYWARD BH}

In this section, we are going to analyse the effect of an extended structure associated with the microscopic discretion of spacetime on the Hayward BH. We plan to consider the noncommutativity to have an intrinsic minimum length scale equal to $\sqrt{\theta}$. Thus, a point like structure will no longer be characterized by a distribution which behaves like Dirac-delta function, but it will be smeared by a distribution of minimal width $\sqrt{\theta}$. Here, $\theta$ can be considered the smallest fundamental cell of an observable area in the deformed theory. Even though the Einstein tensor will not directly get deformed, the deformation of the energy-momentum tensor by an extended structure will induces a deformation of the original Einstein equation. The ordinary classical metric will be recovered at large distances, but new physics will be obtained at short distances, where the effect of an extended structure cannot be neglected.

In the coordinate coherent states approach proposed by Smailagic and Spallucci [58], a point-like mass $M$ instead of being quite localized at a point, is characterized by a smeared structure throughout a region of linear size $\sqrt{\theta}$. The technique we choose here is to seek to a static, spherically symmetric, minimal width, Gaussian distribution of mass whose the noncommutative size is determined by the parameter $\sqrt{\theta}$. Therefore, we should model the mass distributions by a smeared delta function

$$
\rho_{\theta}(r)=\frac{M}{(4 \pi \theta)^{\frac{3}{2}}} e^{-\frac{r^{2}}{4 \theta}} .
$$

The matter density in Eq. (13) displays a physical source which is as close as it is possible to a point-like object. As has been shown in Ref. [63], the noncommutative Hayward mass function $m(r)$ in terms of $g$ and $\theta$ in the metric function (7) can be written as

$$
m(r)=\frac{m_{g} m_{\theta}}{M}
$$

where the Hayward mass function $m_{g}$ in terms of $g$ and the noncommutative mass function 
$m_{\theta}$ in terms of $\theta$ are

$$
\left\{\begin{array}{l}
m_{g}=M\left(\frac{r^{3}}{r^{3}+g^{3}}\right), \\
m_{\theta}=M\left[\mathcal{E}\left(\frac{r}{2 \sqrt{\theta}}\right)-\frac{r}{\sqrt{\pi \theta}} \mathrm{e}^{-\frac{r^{2}}{4 \theta}}\right],
\end{array}\right.
$$

where the Gaussian error function is defined by $\mathcal{E}(x) \equiv \frac{2}{\sqrt{\pi}} \int_{0}^{x} e^{-t^{2}} d t$. In the commutative limit and $g \neq 0$, the function $m(r)$ tends to the Hayward mass function, i.e. $m \rightarrow m_{g}$, while for $\theta \neq 0$ and $g=0$, we get $m \rightarrow m_{\theta}$. This means that for $\theta \rightarrow 0$ and $g \rightarrow 0$, the mass term defined in Eq. (14) is the same as the ADM mass at asymptotic infinity, i.e. $M$, and this reduces to the Schwarzschild case.

An interesting feature of the solution (77) is the horizon equation $N\left(r_{h}\right)=0$. One can draw plots and study the occurrence of horizons. To this purpose, it is convenient to introduce the dimensionless quantities $z=\frac{r}{\sqrt{\theta}}, s=\frac{g}{2 M}$ and $a=\frac{g^{3}}{\theta^{\frac{3}{2}}}$. Therefore the metric function in terms of $z$ turns out to be

$$
N(z)=1-\frac{a^{\frac{1}{3}} z^{2}\left[\mathcal{E}\left(\frac{z}{2}\right)-\frac{z}{\sqrt{\pi}} \mathrm{e}^{-\frac{z^{2}}{4}}\right]}{s\left(z^{3}+a\right)} .
$$

The numerical results of $N(z)$ versus $z$ are presented in Fig. 1, Depending on the values of $a$ and $s$, the metric function (16) displays different causal structures: the entity of two horizons, one horizon (an extremal BH) and no horizon, as can be seen from Fig. (1).

It is important to note that for $z<z_{0}=\frac{r_{0}}{\sqrt{\theta}}$ one cannot speak of an event horizon and no temperature can be defined. The physical description of $r_{0}$ is the smallest radius which cannot be probed by a test particle that is located within some distance from the source, so for $r<r_{0}$ we encounter an unusual dynamical feature leading to, e.g., a negative temperature [65]. In fact, when the $\mathrm{BH}$ reaches the extremal configuration with a minimal radius, the temperature is zero and the Hawking emission abruptly stops. This means that, instead of the ordinary divergent treatment for the ultimate phase of the Hawking evaporation at small radii, there exists a value at which the temperature vanishes. For that reason, the final phase of the $\mathrm{BH}$ evaporation can be considered an extremal $\mathrm{BH}$ relic.

According to Fig. (11), there are two distinct event horizons for $z>z_{0}$. The existence of a minimal nonzero radius, corresponding to the case of an extremal BH configuration $\left(z=z_{0}\right)$, is clear and hence for $z<z_{0}$ there is no event horizon, so that there cannot be a BH. As figure (a) shows, the distance between the horizons increases with decreasing the parameter $s$, while in figure (b), as a increases the distance between two horizons is enlarged. Also, figure (b) implicitly indicates that the minimal nonzero horizon radius increases with raising 
the parameter $a$. We see that the causal structure is more sensitive to the values of $s$, but is roughly unaffected by the values of $a$. This can be rationally explained by the fact that the dimensionless quantity $s$ is dependent on the ADM mass $M$, which plays a key role in the $\mathrm{BH}$ solutions at a broad range of scales, from the short distances to the large distances, while the parameter $a$ is dependent on the parameters which are more important at the short scale gravity.

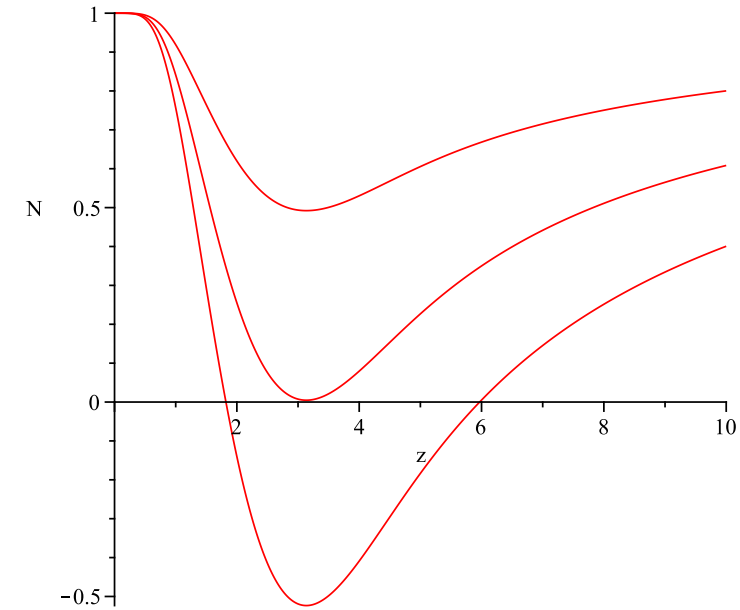

(a)

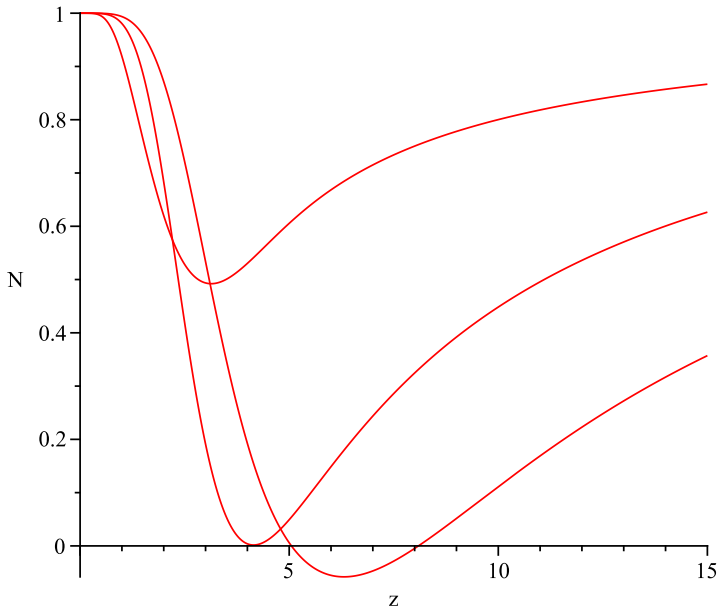

(b)

FIG. 1: $N$ in terms of $z$ for various values of $a$ and $s$. Figures ((a) and (b)) display three possible causal structures: two distinct horizons, one degenerate horizon and no horizon. In figure (a) we have set $a=1.00$. On the right-hand side of figure (a), curves are marked from top to bottom by $s=0.50$ (no horizon), $s=0.25$ (one degenerate horizon) and $s=0.17$ (two distinct horizons) respectively. In figure (b) we have set $s=0.50$. On the right-hand side of figure (b), curves are marked from top to bottom by $a=1.00$ (no horizon), $a=22.42$ (one degenerate horizon) and $a=125.00$ (two distinct horizons) respectively.

The corresponding Lagrangian density for the noncommutative Hayward BH in terms of $g$ and $\theta$ takes the form

$$
L(r)=2 m_{g}\left(\frac{\mathrm{e}^{-\frac{r^{2}}{4 \theta}}}{\sqrt{\pi \theta^{3}}}+\frac{6 g^{3}}{r^{6}} \frac{m}{M}\right) .
$$

In the limit $\theta \rightarrow 0$, one obtains the Lagrangian density for the Hayward $\mathrm{BH}$

$$
L(r)=\frac{12 g^{3}}{r^{6}} \frac{m_{g}^{2}}{M}
$$

and when $g \rightarrow 0$, we have the Lagrangian density for the noncommutative Schwarzschild $\mathrm{BH}$

$$
L(r)=\frac{2 M \mathrm{e}^{-\frac{r^{2}}{4 \theta}}}{\sqrt{\pi \theta^{3}}}=16 \pi \rho_{\theta}(r) .
$$


The role of the parameter $\theta$ is pivotal because it is responsible for the modified causal structure of the solution (compared to the Schwarzschild case) which is similar to the R-N case. For this reason we may call $\theta$ to [66].

There is an important issue here which is worthwhile for discussion. Assuming that the physical treatment of a RBH is qualitatively comparable with or without noncommutativity, a key question has to be asked: is there any motivation to propose the noncommutative's parameter (the fictitious charge) accompanied by the RBH's parameter (the magnetic charge)? In order to provide an accurate answer to this question, it is necessary to point out that there are fundamental distinctions between two situations. First, the noncommutativity is not dependent on the curvature, but is an inherent property of the manifold itself even in the absence of gravity which is denoted by the parameter $\theta$ and can remove some kind of divergences which appear in GR. Thus, if any effect is made by the noncommutativity it must be revealed also in weak fields. Second, the notion of weak or strong field is reasonable only if we compare the field strength with a suitable scale. In GR, there is a natural and unique scale, that is the Planck scale. Hence, the gravitational field strength can still be regarded as weak even near a $\mathrm{BH}$, regarding the Planck scale. This justifies the utilization of linearized field equations as a temporary laboratory to determine the effect of noncommutativity until the horizon radius is larger than the Planck length [67].

On the other hand, the Hayward BH is a regular solution of a modified Einstein equation, and it is also found in a NED field coupled with GR. In the near horizon of a BH, quantum effects are significant due to the strong gravity, thus the spacetime geometry may be modified from quantum effects close to the horizon and the inherent singularity inside the $\mathrm{BH}$ may be removed. With this in mind, we may imagine that the $\mathrm{BH}$ metric is modified close to the horizon region owing to quantum effects. The range of deviations from the standard solution of Einstein equations is determined by free parameters of RBHs. Therefore, free parameters can explain how much quantum effects nearby the horizon influence the deviation from the standard energy level and the radiation. Along this line of reasoning we take the charges as two different issues.

Here, one can expect that a physically reliable NED theory cannot contravene the WEC. Despite the violation of the strong energy condition to have such RBHs, the WEC is still preserved. Apart from some matter fields, the WEC is satisfied by general matter fields. According to Ref. [22], our solution should satisfy the WEC. The WEC expresses that the 
local energy density of matter cannot be negative for all local observers and dominates over the pressure [68]. In other words, the energy density satisfies $T_{\alpha \beta} u^{\alpha} u^{\beta} \geq 0$, where $u^{\alpha}$ is a timelike vector. This is considered to require an anisotropic fluid in order to find a $\mathrm{RBH}$. Thus, the energy-momentum tensor is given by

$$
T_{\beta}^{\alpha}=\operatorname{diag}\left[-\rho(r), p_{r}(r), p_{\perp}(r), p_{\perp}(r)\right]
$$

where $p_{r}=-\rho$ is the radial pressure. So, the energy-momentum tensor confirms the symmetry $T_{0}^{0}=T_{1}^{1}$ as expected. The tangential pressure $p_{\perp}$ is given by

$$
p_{\perp}=-\rho-\frac{r}{2} \partial_{1} \rho
$$

Instead of a point particle, a source turns into a magnetic droplet of anisotropic fluid of density $\rho$. On physical grounds, a non-zero radial pressure is equivalent to preventing droplet to collapse into a matter point. This is an inherent effect on matter owing to the spacetime noncommutativity and it is needed to balance the inward gravitational pull.

The WEC can be expressed as the following inequalities

$$
\left\{\begin{array}{l}
\rho \geq 0 \\
\rho+p_{i} \geq 0 ; \quad i=1,2,3 .
\end{array}\right.
$$

The inequalities above in terms of the mass function can be written as

$$
\left\{\begin{array}{l}
\frac{1}{r^{2}} \frac{d m(r)}{d r} \geq 0, \\
\frac{2}{r} \frac{d m(r)}{d r} \geq \frac{d^{2} m(r)}{d r^{2}},
\end{array}\right.
$$

where

$$
\left\{\begin{array}{l}
\frac{d m(r)}{d r}=m_{g}\left(\frac{r^{2} \mathrm{e}^{-\frac{r^{2}}{4 \theta}}}{2 \sqrt{\pi \theta^{3}}}+\frac{3 g^{3}}{r^{4}} \frac{m}{M}\right) \\
\frac{d^{2} m(r)}{d r^{2}}=\frac{1}{r^{3}+g^{3}}\left[3 g^{3}\left(\frac{1}{r} \frac{d m(r)}{d r}-\frac{m}{r^{2}}-\frac{3 m r}{r^{3}+g^{3}}\right)+\frac{M r^{4} \mathrm{e}^{-\frac{r^{2}}{4 \theta}}}{2 \sqrt{\pi \theta^{3}}}\left(-\frac{3 r^{3}}{r^{3}+g^{3}}-\frac{r^{2}}{2 \theta}+5\right)\right] .
\end{array}\right.
$$

From above relations, it is easy to check that noncommutative Hayward BHs satisfy the WEC everywhere. However, there are some RBH solutions which do not satisfy the WEC [5, 66, 12, 18, 69]. Here we should emphasize that the authors in Ref. [22] have shown that the construction of charged RBH metrics is established upon three requirements: the satisfaction of the WEC, the confirmation of the symmetry $T_{0}^{0}=T_{1}^{1}$ and the asymptotic behavior of the solution as the R-N BH metric. It is evident that our results agree with the mentioned requirements found in [22]. 
It is noteworthy that from the homogeneous equation $R_{\mu \nu}=0$, any vacuum solution must be found only in the absence of matter. However, the above homogeneous equation implicitly presumes that a source term is concentrated on a point at the origin. It seems a kind of physically inconsistent situation that curvature is produced by a zero energy momentum tensor. In any pointless geometry, the implementation of the homogeneous equation, which is dependent upon the notion that matter is concentrated in a single point, is in vain. This is indeed a proper motivation regarding the utility of the noncommutative version of spacetimes. In the noncommutative background, the energy momentum tensor is scattered throughout a region of linear size determined by the parameter $\theta$. Therefore, the virtue of the adopted approach lies in the fact that contrary to other approaches, at very short distances, the regularity of the spacetime is not artificial, but it immediately emerges from the the fluid type picture of the quantum noncommutative fluctuations, while at large distances, NCG turns into a smooth classical manifold.

\section{LAGRANGIAN UNCERTAINTY RELATION}

The HUP is widely considered to be an essential conceptual tool for comprehending differences between classical and quantum theory. On the assumption that the HUP is not always sufficient to confirm the essence of what is non-classical about quantum mechanics, it is sensible to speak of a generalization of it. In other words, a complete formulation of the HUP may provide the quantum essence of quantum theory. For example, the appearance of a minimal observable length is a phenomenological feature of any approach to QG which leads to the GUP in the following form [34]:

$$
\Delta x \Delta p \geq \frac{1}{2}\left(1+\alpha(\Delta p)^{2}+\ldots\right)
$$

where $\alpha$ is a constant of order unity (usually assumed positive) and is dependent on the details of the QG theory. The GUP relation signifies a finite minimal uncertainty $\Delta x_{0}=\sqrt{\alpha}$. Hence, $\Delta x_{0}>0$ may be a manifestation of the fuzzyness of space, or may be considered as a consequence of the smeared structure of the fundamental particles. The above GUP also implies the corresponding corrections to the commutation relation between the momentum operator $\hat{p}$ and the position operator $\hat{x}$ in the pertinent Heisenberg algebra as follows

$$
[\hat{x}, \hat{p}]=i\left(\hat{I}+\alpha \hat{p}^{2}+\ldots\right)
$$


where $\hat{I}$ shows the unit operator. These expressions show that the structure of the spacetime is embellished with an effective minimal length beyond which any measurements to observe a particle location with a precision more than an intrinsic length scale is impossible. The applications and consequences of such a minimal length in a wide range of physical systems have been considered in the literature [70].

In the light of the motivation mentioned above, as a starting point, one can propose a similar idea with Ohanian and Ruffini [55] but conversely to derive a kind of GUP. They utilized the HUP to propose an alternative heuristic derivation of the Hawking radiation. We may calculate a modified Hawking temperature and use the heuristic viewpoint similar to Ref. [55] to find a modified uncertainty principle for radiated photons. On the basis of thermodynamic consistency, BHs emit a thermal black body spectrum at the Hawking temperature,

$$
T_{H}=\left.\frac{1}{4 \pi} \frac{\partial_{1} \mathrm{~g}_{00}}{\sqrt{-\mathrm{g}_{00} \mathrm{~g}_{11}}}\right|_{r=r_{h}}=\left.\frac{1}{4 \pi} \frac{d N(r)}{d r}\right|_{r=r_{h}} .
$$

Using Eqs. (7), (10) and the relation $m\left(r_{h}\right)=\frac{r_{h}}{2}$ at the event horizon, one can easily find the Hawking temperature of noncommutative Hayward BHs as follows

$$
T_{H}=\frac{1}{4 \pi r_{h}}-\frac{r_{h}}{8 \pi} L\left(r_{h}\right)
$$

This temperature leads to a modified uncertainty relation. In this setup, one can estimate the characteristic energy of the emitted photons from the standard uncertainty principle. Near the BH surface, there is an inherent uncertainty in the position of any particle of about the horizon radius $\left(\Delta x \sim r_{h}\right)$, due to the behavior of its field lines [71], and also on dimensional grounds. This leads to the momentum uncertainty in terms of the distance uncertainty and Lagrangian density,

$$
\Delta p \sim \frac{1}{2 r_{h}}-\frac{r_{h}}{4} L\left(r_{h}\right)
$$

and to the energy uncertainty $\Delta E \sim 4 \pi T_{H}$, which is identified as the characteristic energy of the emitted photon or as a characteristic temperature that agrees with the Hawking temperature up to a factor of $4 \pi$ as a calibration factor. From the uncertainty principle, the following modified uncertainty relation including a Lagrangian density function is given by

$$
\Delta x \Delta p \gtrsim \frac{1}{2}\left(1-\frac{(\Delta x)^{2}}{2} L(\Delta x)\right)
$$


The second term on the right-hand side of Eq. (30) is dependent on the parameters of Hayward and noncommutativity. The term of corrections represents the nonlinear magnetic charge and noncommutativity effects of probing photon rather than gravitational effects in comparison with the GUP. The deviation from the standard picture in the solution occurs only in a limited region around the origin. In the limit $\Delta x \gg 1$, the first term on the right-hand side of the modified uncertainty relation is dominant and the HUP is recovered, while in the regime $\Delta x \ll 1$ the second term is dominant and plays an essential role when the momentum and distance scales are in the core around the origin, gravity is actually described by a nonlinear electrodynamic field and the NCG rather than by GR.

The characteristic Lagrangian density of emitted photons is estimated from the HUP. Since $\Delta x$ is associated with the horizon radius, we have $m(\Delta x) \approx \frac{\Delta x}{2}$. Therefore, from Eq. (17) we solve for the Lagrangian uncertainty in terms of the distance uncertainty

$$
\Delta L \approx \frac{2 M}{(\Delta x)^{3}+g^{3}}\left(\frac{(\Delta x)^{3} \mathrm{e}^{-\frac{(\Delta x)^{2}}{4 \theta}}}{\sqrt{\pi \theta^{3}}}+\frac{3 g^{3}}{M(\Delta x)^{2}}\right) .
$$

The Lagrangian uncertainty term emerges from the combined influence of the nonlinear magnetic source and the noncommutativity, such that for $L(\Delta x) \rightarrow 0$ the vacuum solution is recovered that is as $\Delta x \Delta p \geq \frac{1}{2}$. Hence, when $r \rightarrow \infty$ the noncommutative Hayward metric function behaves as the Schwarzschild BH, which implies the HUP.

For further specifications of this issue, the numerical results of the Lagrangian uncertainty versus the distance uncertainty for specific values of $g$ and $\theta$ are presented in Fig. 2 , As the figure shows, for the cases of Hayward and noncommutative Hayward BHs we have roughly similar results at small length scales $(\Delta x \sim 1)$, while the results of noncommutative Schwarzschild and noncommutative Hayward BHs are substantially similar at large length scales $(\Delta x \gg 1)$. However, the results of three cases of $\mathrm{BHs}$ are equivalent at very large distances $(\Delta x \rightarrow \infty)$, which leads to $\Delta L \rightarrow 0$. We can see that the behavior of the noncommutative Schwarzschild case is rather different at very short distances. In the limit $\Delta x \rightarrow 0$, the Lagrangian uncertainty reaches a maximum value for the noncommutative Schwarzschild BH, while it is infinite for two other cases. Nevertheless, as previously stated, we should consider the results just under the circumstance that $r \geq r_{0}$. In other words, there is a minimum uncertainty in position that is equivalent to the minimal nonzero radius, i.e. $\Delta x(\min ) \sim r_{0}$, such that it is impossible to set up a measurement to find a more precise particle position than $\Delta x(\min )$. As a result, the appearance of a lower finite cut off 


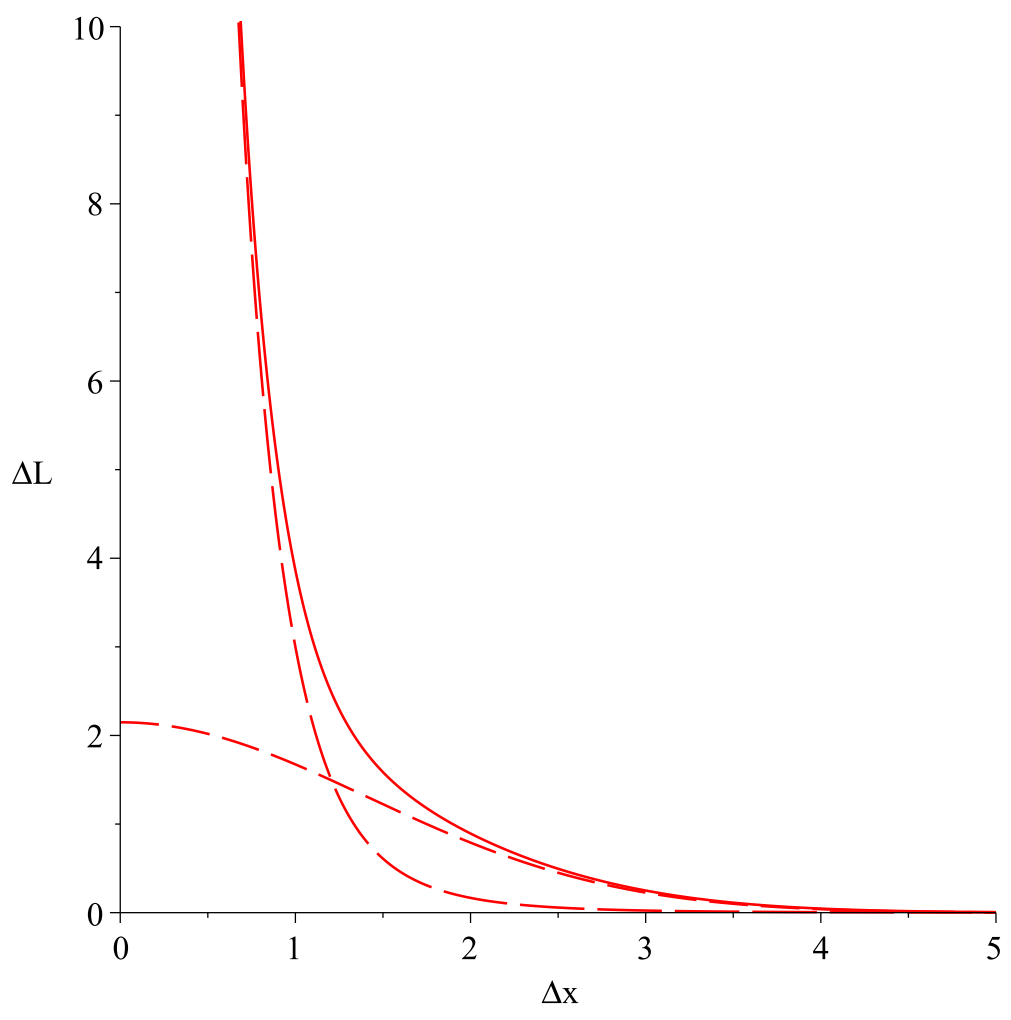

FIG. 2: $\Delta L$ in terms of $\Delta x$ for specific values of $g$ and $\theta$. The solid line corresponds to the noncommutative Hayward BH for $g=1.00$ and $\theta=1.00$. The dash line refers to the noncommutative Schwarzschild BH for $g=0$ and $\theta=1.00$. The long dash line represents the Hayward BH for $g=1.00$ and $\theta=0$. We have set $M=M(\min )$ for each cases.

at the short scale gravity compels a bound on any measurements to determine a particle position in a noncommutative gravity theory. Therefore, one should set the requirement that $L(\Delta x) \lesssim \Delta L(\max )$ because for the pattern of the metric for $\Delta L>\Delta L(\max )$ no sensible temperature can be determined. This upper bound on the Lagrangian uncertainty is a necessary condition for preventing the unusual thermodynamical behavior at very short distances. In Table I, the numerical results of the minimum distance uncertainty, minimum mass and maximum Lagrangian uncertainty for specific values of $g$ and $\theta$ are presented. As the table shows, the nonlinear electrodynamic field and/or the fictitious charge lead to the existence of a remnant mass $\left(M_{0}\right)$ in which the $\mathrm{BH}$ can shrink to. The results are confirmed by the numerical solutions of Ref. [63] for the three models of nonsingular BHs. This means that the existence of the fictitious-magnetic charge, fictitious charge and magnetic charge are responsible for the nonsingular solutions in the noncommutative Hayward $\mathrm{BH}$, noncommutative Schwarzschild $\mathrm{BH}$ and Hayward $\mathrm{BH}$, respectively. As a final note, if we 
TABLE I: This table shows the remnant radius, remnant mass and maximum Lagrangian uncertainty for specific values of $g$ and $\theta$ that correspond to various kinds of BHs and hence are in agreement with Fig. 2

\begin{tabular}{|c|c|c|}
\hline Noncommutative Hayward BH & Noncommutative Schwarzschild BH & Hayward BH \\
\hline$\Delta x(\min ) \sim r_{0} \approx 3.13$ & $\Delta x(\min ) \sim r_{0} \approx 3.02$ & $\Delta x(\min ) \sim r_{0} \approx 1.26$ \\
\hline$M(\min ) \sim M_{0} \approx 1.97$ & $M(\min ) \sim M_{0} \approx 1.90$ & $M(\min ) \sim M_{0} \approx 0.94$ \\
\hline$\Delta L(\max ) \approx 0.20$ & $\Delta L(\max ) \approx 0.22$ & $\Delta L(\max ) \approx 1.26$ \\
\hline
\end{tabular}

had chosen the Bardeen solution, as another popular example of RBHs, despite that only the mass function would have altered, the general properties would have directed to wholly equivalent results to those above [62].

\section{CONCLUSIONS}

In summary, we have investigated a general ansatz of the BH solution having the static spherical symmetric configuration with a fictitious charge and the nonlinear magnetic charge in the context of Einstein-NED theory. We have studied the Hawking temperature of noncommutative Hayward BHs. In this way, a modified uncertainty relation is achieved in a heuristic way by the utilization of the Hawking temperature and general properties of BHs according to Ref. [55]. The term of corrections is due to the Hayward's parameter, stemming from a nonlinear electrodynamic field, plus noncommutativity effects. As a result, the modified uncertainty relation including a Lagrangian density function is emerged from nonlinear electromagnetism as a physical source and an inherent property of the manifold instead of gravitational effects compared to the GUP. Ultimately, it is found that there is an upper bound on the Lagrangian uncertainty of BHs at the short scale gravity which comes from the deSitter core around the origin.

The authors in Ref. [72] have recently shown that the behavior of Hawking radiation close to the Planck phase is significantly modified in the framework of gravity's rainbow. This modification gives corrections to the thermodynamic description of BHs and predicts the existence of $\mathrm{BH}$ remnant. As a potential problem, it will be interesting to investigate the behavior of Hawking radiation for the noncommutative inspired Hayward BH through 
rainbow functions.

In addition, it is important to point out the very recent study on the noncommutative inspired electrically and magnetically charged BH in Euler-Heisenberg NED model [73]. In this model, the WEC was examined and satisfied in opposition to the commutative case. In their setup, the formation of shadows for the noncommutative inspired metrics was addressed and also it was shown that the modification due to the noncommutativity might be susceptible to observation using new generation interferometers. It would also be interesting to extend the present analysis to the Euler-Heisenberg NED model.

Finally, it is interesting to note that the Cauchy horizons of the R-N BHs [74], Schwarzschild white holes [75] and Schwarzschild wormholes [76] are unstable. In general, an observer crossing the Cauchy horizon might encounter an arbitrarily large blue shift of any incoming radiation; so that any small perturbation might disrupt the horizon and result in a curvature singularity. In what concerns the possible blue shift instability at the inner Cauchy horizon of a noncommutative inspired BH, one should emphasize that the curvature singularity is relevant only for a classical differential manifold. The propagation of any field in a noncommutative spacetime is under the influence of a natural ultraviolet cutoff in which an infinite amount of energy near the inner Cauchy horizon cannot be observed. On the other hand, the violation of the strong energy condition could occur outside the inner horizon wherein gravity is actually described by NCG and not by GR. Thereby, the situation for noncommutative inspired BHs is quite different and we leave it as an open problem that goes beyond the scope of the present paper. We hope to further investigate this issue in future work.

[1] R. Pelliger and R. Torrence, J. Math. Phys. 10, 1718 (1969)

[2] K. A. Bronnikov and G. N. Shikin, "On the Reissner-Nordström problem with a nonlinear electromagnetic field". In: "Classical and Quantum Theory of Gravity", Trudy IF AN BSSR, p. 88, Minsk 1976 (in Russian)

[3] K. A. Bronnikov, V. N. Melnikov, G. N. Shikin and K. P. Staniukovich, Ann. Phys. (USA) 118, $84(1979)$

[4] E. Ayón-Beato and A. García, Phys. Rev. Lett. 80, 5056 (1998), arXiv:gr-qc/9911046 
[5] E. Ayón-Beato and A. García, Phys. Lett. B 464, 25 (1999), arXiv:hep-th/9911174

[6] E. Ayón-Beato and A. García, Gen. Relativ. Gravit. 31, 629 (1999), arXiv:gr-qc/9911084

[7] E. S. Fradkin and A. A. Tseytlin, Phys. Lett. B 163, 123 (1985)

[8] A. A. Tseytlin, Nucl. Phys. B 276, 391 (1986)

[9] N. Seiberg and E. Witten, JHEP 9909, 032 (1999), arXiv:hep-th/9908142

[10] See for a review, S. Ansoldi, in Conference Proceedings of BH2 (Italy, Milano, May 10-12 2007), arXiv:0802.0330

[11] J. M. Bardeen, in Conference Proceedings of GR5 (Tbilisi, USSR, 1968), p. 174

[12] K. A. Bronnikov, Phys. Rev. D 63, 044005 (2001), arXiv:gr-qc/0006014

[13] S. A. Hayward, Phys. Rev. Lett. 96, 031103 (2006), arXiv:gr-qc/0506126

[14] E. Ayón-Beato and A. García, Phys. Lett. B 493, 149 (2000), arXiv:gr-qc/0009077

[15] Z-Y. Fan, Eur. Phys. J. C 77, 266 (2017), arXiv:1609.04489

[16] Z-Y. Fan and X. Wang, Phys. Rev. D 94, 124027 (2016), arXiv:1610.02636

[17] B. Toshmatov, Z. Stuchlík and B. Ahmedov, Phys. Rev. D 95, 084037 (2017), arXiv:1704.07300

[18] E. Ayón-Beato and A. García, Gen. Rel. Grav. 37, 635 (2005), arXiv:hep-th/0403229

[19] K. A. Bronnikov and J. C. Fabris, Phys. Rev. Lett. 96, 251101 (2006), arXiv:gr-qc/0511109

[20] W. Berej, J. Matyjasek, D. Tryniecki and M. Woronowicz, Gen. Rel. Grav. 38, 885 (2006), arXiv:hep-th/0606185

[21] M. Azreg-Aïnou, Phys. Lett. B 730, 95 (2014), arXiv:1401.0787

[22] L. Balart and E. C. Vagenas, Phys. Lett. B 730, 14 (2014), arXiv:1401.2136]

[23] I. Radinschi, F. Rahaman, T. Grammenos, A. Spanou and S. Islam, Adv. Math. Phys. 2015, 530281 (2015), arXiv:1404.6410

[24] E. L. B. Junior, M. E. Rodrigues, and M. J. S. Houndjo, J. Cosmol. Astropart. Phys. 10, 060 (2015), arXiv:1503.07857

[25] I. Dymnikova and E. Galaktionov, Class. Quant. Grav. 32, 165015 (2015), arXiv:1510.01353.

[26] E. Chaverra, J. C. Degollado, C. Moreno and O. Sarbach, Phys. Rev. D 93, 123013 (2016), arXiv:1605.04003

[27] K. A. Bronnikov, Phys. Rev. D 96, 128501 (2017), arXiv:1712.04342

[28] K. A. Bronnikov, Int. J. Mod. Phys. D 27, 1841005 (2018), arXiv:1711.00087

[29] M.-S. Ma, Ann. Phys. 362, 529 (2015), arXiv:1509.05580; I. Dymnikova, Int. J. Mod. Phys. 
D 24, 1550094 (2015), arXiv:1510.01352]; A. Sheykhi and F. Shaker, Phys. Lett. B 754, 281 (2016), arXiv:1601.04035]; S. H. Mazharimousavi, Z. Amirabi and M. Halilsoy, Gen Rel. Grav. 48, 143 (2016), arXiv:1703.05316]; A. Övgün, G. Leon, J. Magaña and K. Jusufi, Eur. Phys. J. C 78, 462 (2018), arXiv:1709.09794]; B. Toshmatov, Z. Stuchlík, J. Schee and B. Ahmedov, Phys. Rev. D 97, 084058 (2018), arXiv:1805.00240; X.-M. Kuang, B. Liu and A. Övgün, Eur. Phys. J. C 78, 840 (2018), arXiv:1807.10447]; B. Toshmatov, Z. Stuchlík and B. Ahmedov, Phys. Rev. D 98, 085021 (2018), arXiv:1810.06383]; B. Toshmatov, Z. Stuchlík, B. Ahmedov and D. Malafarina, Phys. Rev. D 99, 064043 (2019), arXiv:1903.03778

[30] S. W. Hawking and G. Ellis, The Large Scale Structure of Space-Time, Cambridge University Press, Cambridge, 1973

[31] P. Nicolini, Int. J. Mod. Phys. A 24, 1229 (2009), arXiv:0807.1939]

[32] H. Hinrichsen and A. Kempf, J. Math. Phys. 37, 2121 (1996), arXiv:hep-th/9510144; A. Kempf, J. Math. Phys. 38, 1347 (1997), arXiv:hep-th/9602085

[33] D. Amati, M. Ciafaloni and G. Veneziano, Int. J. Mod. Phys. A 3, 1615 (1988)

[34] A. N. Tawfik and A. M. Diab, Rept. Prog. Phys. 78, 126001 (2015); Int. J. Mod. Phys. D 23, 1430025 (2014), arXiv:1410.0206]

[35] S. Gangopadhyay, A. Dutta and A. Saha, Gen. Rel. Grav. 46, 1661 (2014), arXiv:1307.7045.

[36] D. N. Page, Phys. Rev. Lett. 71, 3743 (1993) arXiv:hep-th/9306083

[37] P. Chen, Y. C. Ong and D.-h. Yeom, Physics Reports 1-45 (2015) arXiv:1412.8366]

[38] S. W. Hawking, Commun. Math. Phys. 43, 199 (1975)

[39] A. Strominger, Les Houches lectures on BHs, arXiv:hep-th/9501071

[40] M. K. Parikh and F. Wilczek, Phys. Rev. Lett. 85, 5042 (2000), arXiv:hep-th/9907001

[41] M. K. Parikh, Int. J. Mod. Phys. D 13, 2351 (2004), arXiv:hep-th/0405160

[42] K. Srinivasan, T. Padmanabhan, Phys. Rev. D 60, 024007 (1999), arXiv:gr-qc/9807064

[43] F. S. Mirekhtiary and I. Sakalli, Teor. Mat. Fiz. 198, 455 (2019); K. Jusufi, I. Sakalli and A. Övgün, Gen. Rel. Grav. 50, 10 (2018); A. Övgün and I. Sakalli, Int. J. Theor. Phys. 57, 322 (2018); I. Sakalli, K. Jusufi and A. Övgün, Gen. Rel. Grav. 50, 125 (2018); K. Jusufi and A. Övgün, Int. J. Theor. Phys. 56, 1725 (2017); I. Sakalli and A. Övgün, Europhys. Lett. 118, 60006 (2017); X. M. Kuang, J. Saavedra and A. Övgün, Eur. Phys. J. C 77, 613 (2017); I. Sakalli and A. Övgün, Eur. Phys. J. Plus 131, 184 (2016); J. Astrophys. Astron. 37, 21 (2016); J. Exp. Theor. Phys. 121, 404 (2015); Astrophys. Space Sci. 359, 32 (2015); 
Europhys. Lett. 110, 10008 (2015); I. Sakalli, Astrophys. Space Sci. 340, 317 (2012); I. Sakalli, M. Halilsoy and H. Pasaoglu, Astrophys. Space Sci. 340, 155 (2012); Int. J. Theor. Phys. 50, 3212 (2011); S. H. Mehdipour, Int. J. Mod. Phys. A 25, 5543 (2010), arXiv:1004.1255; Phys. Rev. D 81, 124049 (2010), arXiv:1006.5215]; K. Nozari and S. H. Mehdipour, JHEP 03, 061 (2009), arXiv:0902.1945]; H. Pasaoglu and I. Sakalli, Int. J. Theor. Phys. 48, 3517 (2009); S. H. Mazharimousavi, I. Sakalli and M. Halilsoy, Phys. Lett. B 672, 177 (2009); K. Nozari and S. H. Mehdipour, Class. Quantum Grav. 25, 175015 (2008), arXiv:0801.4074]; Europhys. Lett. 84, 20008 (2008), arXiv:0804.4221

[44] E. P. Verlinde, JHEP 1104, 029 (2011), arXiv:1001.0785.

[45] P. Chen and C.-H. Wang, arXiv:1112.3078]

[46] A. F. Ali and A. Tawfik, Adv. High Energy Phys. 2013, 126528 (2013), arXiv:1301.3508

[47] Z.-W. Feng, S.-Z. Yang, H.-L. Li and X.-T. Zu, Phys. Lett. B 768, 81 (2017), arXiv:1610.08549

[48] B. P. Abbott et al. (LIGO Scientific Collaboration and Virgo Collaboration), Phys. Rev. Lett. 116, 061102 (2016), arXiv:1602.03837]

[49] J. R. Mureika, Phys. Lett. B 789, 88 (2019)

[50] (The Event Horizon Telescope collaboration), http://www.eventhorizontelescope.org/

[51] G. Lambiase and F. Scardigli, Phys. Rev. D 97, 075003 (2018), arXiv:1709.00637]

[52] S. W. Hawking, Phys Rev D 14, 2460 (1976)

[53] Y. S. Myung, Y-W. Kim and Y-J. Park, Phys. Lett. B 645, 393 (2007), arXiv:gr-qc/0609031]; W. Kim, E. J. Son and M. Yoon, JHEP 0801, 035 (2008), arXiv:0711.0786]; L. Xiang and X. Q. Wen, JHEP 0910, 046 (2009), arXiv:0901.0603]; R. Banerjee and S. Ghosh, Phys. Lett. B 688, 224 (2010), arXiv:1002.2302]; R. V. Maluf and J. C. S. Neves, Phys. Rev. D 97, 104015 (2018), arXiv:1801.02661

[54] R. J. Adler, P. Chen and D. I. Santiago, Gen. Rel. Grav. 33, 2101 (2001), arXiv:gr-qc/0106080

[55] H. Ohanian and R. Ruffini, Gravitation and Spacetime, 2nd ed., p. 481 (W. W. Norton, 1994)

[56] A. J. M. Medved and E. C. Vagenas, Phys. Rev. D 70, 124021 (2004), arXiv:hep-th/0411022; B. Bolen and M. Cavaglia, Gen. Rel. Grav. 37, 1255 (2005), arXiv:gr-qc/0411086; K. Nozari and S. H. Mehdipour, Mod. Phys. Lett. A 20, 2937 (2005), arXiv:0809.3144; Int. J. Mod. Phys. A 21, 4979 (2006), arXiv:gr-qc/0511110; B. J. Carr, J. Mureika and P. Nicolini, JHEP 1507, 052 (2015), arXiv:1504.07637. 
[57] D. Amati, M. Ciafaloni and G. Veneziano, Phys. Lett. B 216, 41 (1989); M. Maggiore, Phys. Rev. D 49, 5182 (1994), arXiv:hep-th/9305163; M. Sprenger, P. Nicolini and M. Bleicher, Eur. J. Phys. 33, 853 (2012), arXiv:1202.1500

[58] A. Smailagic and E. Spallucci, J. Phys. A 36, L467 (2003), arXiv:hep-th/0307217]; 36, L517 (2003) arXiv:hep-th/0308193]; 37, 7169 (2004), arXiv:hep-th/0406174

[59] F. Briscese, E. R. Bezerra de Mello, A. Yu. Petrov and V. B. Bezerra, Phys. Rev. D 92, 104026 (2015), arXiv:1508.02001

[60] M. Maggiore, Phys. Lett. B 304, 65 (1993), arXiv:hep-th/9301067

[61] M. Rizwan, M. Z. Ali and A. Övgün, Mod. Phys. Let. A 33, 1950184 (2019), arXiv:1812.01983; W. Javed, R. Babar and A. Övgün, Mod. Phys. Let. A 34, 1950057 (2019), arXiv:1808.09795; P. A. González, A. Övgün, J. Saavedra and Y. Vásquez, Gen. Rel. Grav. 50, 62 (2018), arXiv:1711.01865; A. Övgün and K. Jusufi, Eur. Phys. J. Plus 132, 298 (2017), arXiv:1703.08073]; Eur. Phys. J. Plus 131, 177 (2016), arXiv:1512.05268]; I. Sakalli, A. Övgün and K. Jusufi, Astrophys. Space Sci. 361, 330 (2016), arXiv:1602.04304; A. Övgün, Int. J. Theor. Phys. 55, 2919 (2016), arXiv:1508.04100; I. Sakalli and A. Övgün, Gen. Rel. Grav. 48, 1 (2016), arXiv:1507.01753]; Eur. Phys. J. Plus 130, 110 (2015), arXiv:1505.02093; V. Akhmedova, T. Pilling, A. de Gill and D. Singleton, Phys. Lett. B 666, 269 (2008), arXiv:0804.2289]; E. T. Akhmedov, V. Akhmedova and D. Singleton, Phys. Lett. B 642, 124 (2006), [arXiv:hep-th/060809]

[62] S. H. Mehdipour and M. H. Ahmadi, Astrophys. Space Sci. 361, 314 (2016), arXiv:1604.06272

[63] S. H. Mehdipour and M. H. Ahmadi, Nucl. Phys. B 926, 49 (2018), arXiv:1604.08584]

[64] K. A. Bronnikov, Grav. Cosmol. 23, 343 (2017), arXiv:1708.08125,

[65] K. Nozari, S. H. Mehdipour, Chaos Solitons Fractals 39, 956 (2009), arXiv:hep-th/0610076; S. H. Mehdipour, Eur. Phys. J. Plus 127, 80 (2012), arXiv:1111.2468]; Eur. Phys. J. Plus 129, 197 (2014), arXiv:1408.4772

[66] P. Nicolini and G. Torrieri, JHEP 1108, 097 (2011), arXiv:1105.0188,

[67] P. Nicolini, J. Phys. A 38, L631 (2005), arXiv:hep-th/0507266

[68] E. Poisson, "A relativist's toolkit: the mathematics of black hole mechanics", Cambridge University Press, 2004.

[69] A. Burinskii and S. R. Hildebrandt, Phys. Rev. D 65, 104017 (2002), arXiv:hep-th/0202066

[70] E. C. Vagenas, A. F. Ali and H. Alshal, Eur. Phys. J. C 79, 276 (2019); S. Kanzi and I. 
Sakalli, Nucl. Phys. B 946, 114703 (2019); A. Iorio et al., Int. J. Mod. Phys. D 27, 1850080 (2018); M. C. Braidotti et al., Europhys. Lett. 124, 44001 (2018); G. Gecim and Y. Sucu Gen. Rel. Grav. 50, 152 (2018); Y. Gim, H. Um and W. Kim, Phys. Lett. B 784, 206 (2018); Q. Zhao, M. Faizal and Z. Zaz, Phys. Lett. B 770, 564 (2017); M. Khodadi, K. Nozari and A. Hajizadeh, Phys. Lett. B 770, 556 (2017); L. B. Castro and A. E. Obispo, J. Phys. A: Math. Theor. 50, 285202 (2017); A. Bhat, S. Dey, M. Faizal, C. Hou and Q. Zhao, Phys. Lett. B 770, 325 (2017); M. Faizal and T. S. Tsun, Nucl. Phys. B 924, 588 (2017); M. Salah, F. Hammad, M. Faizal and A. F. Ali, JCAP 02, 035 (2017); M. Faizal, A. F. Ali and A. Nassar, Phys. Lett. B 765, 238 (2017)

[71] R. J. Adler and T. K. Das, Phys. Rev. D 14, 2472 (1976)

[72] A. F. Ali, Phys. Rev. D 89, 104040 (2014), arXiv:1402.5320]; A. F. Ali, M. Faizal and M. M. Khalil, Phys. Lett. B 743, 295 (2015), arXiv:1410.4765]; A. F. Ali, M. Faizal and M. M. Khalil, Nucl. Phys. B 894, 341 (2015), arXiv:1410.5706]

[73] M. Maceda and A. Macías, Phys. Lett. B 788, 446 (2019)

[74] J. M. McNamara, Proc. R. Soc. London A 358, 499 (1978)

[75] D. M. Eardley, Phys. Rev. Lett. 33, 442 (1974)

[76] I. Redmount, Prog. of Theor. Phys. 73, 1401 (1985) 